\title{
Optical characterization of gold-cuprous oxide interfaces for terahertz emission applications
}

\author{
Gopika K. P. Ramanandan, ${ }^{1, \star}$ Aurèle J. L. Adam, ${ }^{1}$ Gopakumar Ramakrishnan, ${ }^{1}$ \\ Peter Petrik, ${ }^{1,2}$ Ruud Hendrikx, ${ }^{3}$ and Paul C. M. Planken ${ }^{1}$ \\ 'Optics Research Group, Faculty of Applied Physics, Delft University of Technology, \\ Lorentzweg 1, 2628 CJ Delft, The Netherlands \\ ${ }^{2}$ Research Institute for Technical Physics and Materials Science, H-1525 Budapest, P.O. Box 49, Hungary \\ ${ }^{3}$ Department of Materials Science and Engineering, Delft University of Technology, \\ Mekelweg 2, 2628 CD Delft, The Netherlands \\ ${ }^{*}$ Corresponding author: g.r.kottayipilappara@ tudelft.nl
}

Received 29 November 2013; revised 20 February 2014; accepted 20 February 2014; posted 20 February 2014 (Doc. ID 201874); published 24 March 2014

\begin{abstract}
We show that the interface between gold and thermally formed cuprous oxide, which emits terahertz radiation when illuminated with ultrafast femtosecond lasers, is in fact an $\mathrm{AuCu} / \mathrm{Cu}_{2} \mathrm{O}$ interface due to the formation of the thermal diffusion alloy $\mathrm{AuCu}$. The alloy enables the formation of a Schottkybarrier-like electric field near the interface which is essential to explain the $\mathrm{THz}$ emission from these samples. We confirm the formation of this $\mathrm{AuCu}$ layer by x-ray diffraction measurements, ellipsometry, and visual inspection. We determined the frequency-dependent complex refractive indices of the $\mathrm{Cu}_{2} \mathrm{O}$ and $\mathrm{AuCu}$ layer and verified them using reflection spectroscopy measurements. These refractive indices can be used for optimizing the thickness of $\mathrm{Cu}_{2} \mathrm{O}$ for maximum $\mathrm{THz}$ emission from these interfaces. (c) 2014 Optical Society of America
\end{abstract}

OCIS codes: $\quad$ (310.6860) Thin films, optical properties; (300.6495) Spectroscopy, terahertz; (310.6870)

Thin films, other properties; (120.2130) Ellipsometry and polarimetry.

http://dx.doi.org/10.1364/AO.53.001994

\section{Introduction}

Many metal/semiconductor interfaces emit $\mathrm{THz}$ radiation when excited with femtosecond laser pulses $[1,2]$. The laser pulses optically generate charge carriers in the semiconductor, and the $\mathrm{THz}$ emission is the result of carrier acceleration by the electric depletion field that can naturally form near a $\mathrm{metal} /$ semiconductor interface. In general, the $\mathrm{THz}$ emission is weak when the metal is deposited on top of the semiconductor. This poor $\mathrm{THz}$ generation efficiency results from the power loss of the pump laser pulses and of the generated $\mathrm{THz}$ pulses in the

$1559-128 \mathrm{X} / 14 / 101994-07 \$ 15.00 / 0$

(C) 2014 Optical Society of America metal film and from the fact that light is also absorbed in a region of the semiconductor where there is no depletion field. Recently, we showed that the $\mathrm{THz}$ emission of inverted structures, ultrathin semiconductor layers deposited on gold, is much stronger [3]. When an optimum thickness of the semiconductor layer is chosen, multiple reflections occur inside the layer which acts as an antireflection coating, increasing the absorption within the semiconducting thin film. This effect, also known as cavity-enhanced optical absorption [4], ensures that the maximum absorption occurs in the Schottky-depletion layer, where it matters for the $\mathrm{THz}$ generation. One of the surprising results of this work is that cuprous oxide $\left(\mathrm{Cu}_{2} \mathrm{O}\right)$ thin films deposited on gold substrates emit strong $\mathrm{THz}$ pulses in this manner. The emission 


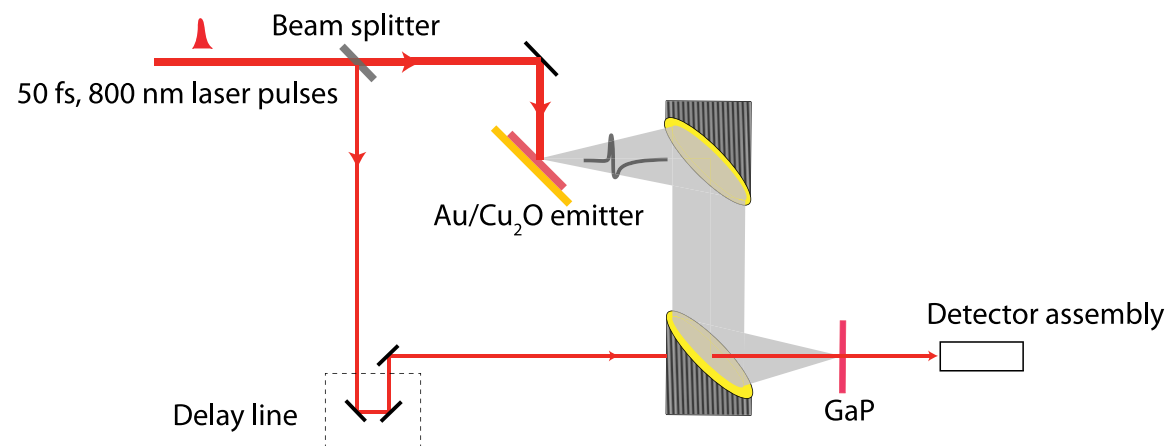

Fig. 1. THz generation setup. Ultrafast laser pulses are incident on an $\sim 400 \mathrm{~nm}$ thick $\mathrm{Cu}_{2} \mathrm{O}$ film deposited on a Au substrate at $45^{\circ}$ angle of incidence. Parabolic mirrors are used to collect, collimate, and focus the emitted THz pulses onto a GaP electro-optic crystal. A probe laser beam is also focused onto the GaP crystal. The detector assembly measures the instantaneous $\mathrm{THz}$ electric field in the GaP detection crystal as a change in the polarization state of the probe laser beam.

is particularly unexpected because the interfaces formed by depositing $\mathrm{Au}$ on $\mathrm{Cu}_{2} \mathrm{O}$ are ohmic junctions rather than Schottky junctions [5].

Here, we show optical (and x ray) measurements of the interface layer between $\mathrm{Au}$ and $\mathrm{Cu}_{2} \mathrm{O}$ which demonstrate that oxidation of $\mathrm{Cu}$ on $\mathrm{Au}$ leads to the formation of a $\mathrm{CuAu}$ alloy and to the formation of a Schottky-like barrier between the alloy and the $\mathrm{Cu}_{2} \mathrm{O}$. We have determined the frequencydependent complex refractive indices of this alloy and also of the thin $\mathrm{Cu}_{2} \mathrm{O}$ film using variable-angle spectroscopic ellipsometry. The reflection spectra, calculated with these refractive index values, agree well with the measurements. The optical data is particularly interesting and useful for the optimization of the optical absorption and $\mathrm{THz}$ emission from these interfaces.

\section{Terahertz Generation}

Gold substrates are prepared by electron-beam evaporation of $\mathrm{Au}$ onto a clean silicon wafer in vacuum. A thin film of chromium is also evaporated onto the silicon substrate prior to the deposition of gold in order to improve the adhesion of gold to the silicon substrate. The chromium and gold layers are each $50 \mathrm{~nm}$ thick. A thin film of copper is then evaporated onto the gold substrate. The Cu thin film is then completely oxidized by thermal oxidation in ambient air at a temperature of $220^{\circ} \mathrm{C}$ to get $\mathrm{Cu}_{2} \mathrm{O}$ thin films. The thickness of the $\mathrm{Cu}_{2} \mathrm{O}$ thin film formed after oxidation is about 1.5 times that of the original copper thin film. Although copper can form at least two types of stable oxides, it has been observed that for temperatures lower than $250^{\circ} \mathrm{C}, \mathrm{Cu}_{2} \mathrm{O}$ is predominantly formed $[6,7]$.

The experimental setup used for our $\mathrm{THz}$ generation measurements is schematically shown in Fig. 1. We use $50 \mathrm{fs}$ laser pulses from a Ti:sapphire laser centered at a wavelength of $800 \mathrm{~nm}$. The average power of the laser source is $2.5 \mathrm{~W}$ and the repetition rate is $5.2 \mathrm{MHz}$. The laser beam is split into pump and probe beams by a 90:10 beam splitter. $90 \%$ of the laser power is used for the generation of $\mathrm{THz}$ pulses from the $\mathrm{Au} / \mathrm{Cu}_{2} \mathrm{O}$ thin films. The samples are mounted in a reflection-type configuration, and the laser is incident on the sample at an angle of $45^{\circ}$. The pump pulses are delayed using a retroreflecting mirror arrangement mounted on a translation stage. The generated $\mathrm{THz}$ pulses are collected, collimated, and focused onto a $300 \mu \mathrm{m}$ GaP crystal which acts as an electro-optic detection crystal [8]. The probe beam is also focussed onto the detection crystal through an aperture in the last parabolic mirror. The electric field of the $\mathrm{THz}$ pulse modifies the polarization state of the $800 \mathrm{~nm}$ probe beam which is measured using an arrangement of a quarter-wave plate, a Wollaston prism, and a differential detector. The setup is purged with dry nitrogen gas in order to reduce the absorption by water vapor in the atmosphere. The spectrum of the measured $\mathrm{THz}$ pulse is obtained by Fourier-transforming the timedependent $\mathrm{THz}$ electric field.

The electric field emitted by a $420 \mathrm{~nm}$ thick $\mathrm{Cu}_{2} \mathrm{O}$ thin film on gold and the corresponding Fouriertransformed spectrum are shown in Fig. 2. The bandwidth of the emitted $\mathrm{THz}$ pulse ranges from 0.1 to almost $7.5 \mathrm{THz}$. Interestingly, the TO phonon resonance of $\mathrm{Cu}_{2} \mathrm{O}$ at $\sim 4.5 \mathrm{THz}$ [9] can be seen in the $\mathrm{THz}$ emission spectrum, indicating that the $\mathrm{Cu}_{2} \mathrm{O}$ thin film is polycrystalline in nature.

\section{Interfacial Alloy Formation}

The most plausible explanation for $\mathrm{THz}$ generation from $\mathrm{Au} / \mathrm{Cu}_{2} \mathrm{O}$ junctions is carrier acceleration by the Schottky barrier electric field near the $\mathrm{Au} / \mathrm{Cu}_{2} \mathrm{O}$ interface. However, earlier reports indicate that the junctions that form when $\mathrm{Au}$ is deposited on $\mathrm{Cu}_{2} \mathrm{O}$ are essentially ohmic in nature [5]. In order to verify the Schottky junction formation when $\mathrm{Cu}_{2} \mathrm{O}$ thin films are fabricated on gold substrates, we measured the current voltage (I-V) characteristics of one of the samples. The measured I-V characteristic of such a sample is shown by the solid red line in Fig. 3 . We can see that the junction formed by the oxidation of a $\mathrm{Cu}$ film deposited on $\mathrm{Au}$ has a nonlinear $\mathrm{I}-\mathrm{V}$ curve. We also made a sample where, first, the $\mathrm{Cu}$ was deposited and oxidized to $\mathrm{Cu}_{2} \mathrm{O}$, followed by the deposition of $\mathrm{Au}$. The junction formed by this 

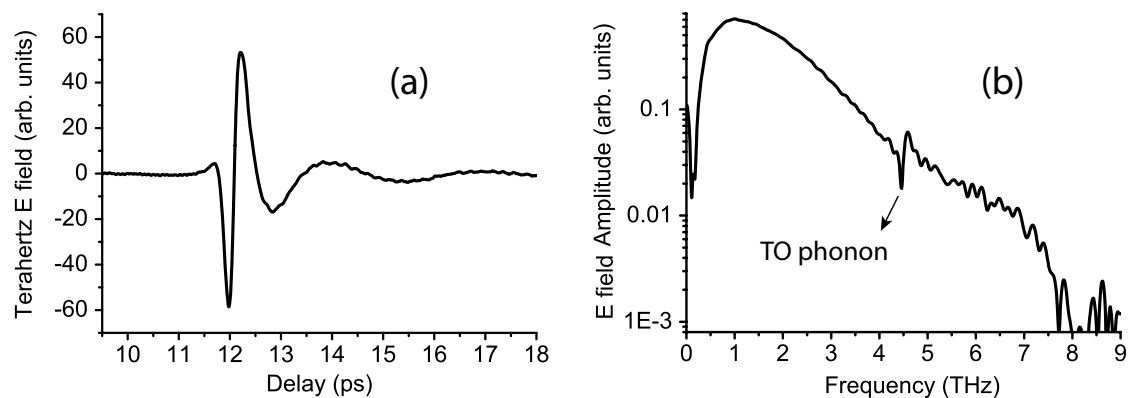

Fig. 2. (a) Time-dependent electric field of the THz pulse emitted from the Au- $\mathrm{Cu}_{2} \mathrm{O}$ Schottky junction and (b) the corresponding Fouriertransformed spectrum.

sample is characterized by a linear I-V curve (dashed line in Fig. 3), typical of an ohmic junction. This interesting result can be explained as follows. The barrier height of a Schottky interface depends on the work function of the metal. The samples used for terahertz emission experiments are thermally formed $\mathrm{Cu}_{2} \mathrm{O}$ thin films on gold substrates. During the process of heating copper to get the oxide film, inter-metallic compounds or alloys of $\mathrm{Au} / \mathrm{Cu}$ can be formed by diffusion [10]. In our experiments, this would mean that the interface we have is not an $\mathrm{Au}-\mathrm{Cu}_{2} \mathrm{O}$ interface, but an $\mathrm{AuCu}-\mathrm{Cu}_{2} \mathrm{O}$ interface. This can cause the junction characteristics to differ from the one with a pure $\mathrm{Au} / \mathrm{Cu}_{2} \mathrm{O}$ interface. Note that although the nonlinearity of the $\mathrm{I}-\mathrm{V}$ curve is indicative of a barrier formation, a deviation from the ideal behavior of a Schottky diode is observed. The nearly point-symmetric nature of the observed $\mathrm{I}-\mathrm{V}$ curve is currently under investigation.

In order to investigate the presence of such a diffusion layer, we removed the thermal oxide film from the gold substrate by treating it with dilute nitric acid. A color contrast-enhanced photograph of a pure gold substrate and a gold substrate after removing the $\mathrm{Cu}_{2} \mathrm{O}$ film is shown in Fig. $\underline{4}$. The surface of

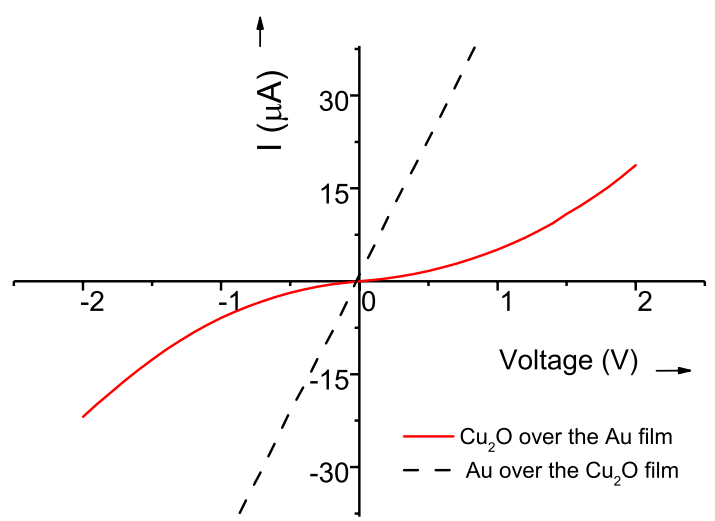

Fig. 3. Measured I-V characteristics of the $\mathrm{Au} / \mathrm{Cu}_{2} \mathrm{O}$ junctions. The solid line shows the junction characteristics of a $\mathrm{Cu}_{2} \mathrm{O}$ thin film fabricated over a Au thin film substrate and the dashed line shows the same when the Au thin film is deposited over the $\mathrm{Cu}_{2} \mathrm{O}$ thin film. The difference in the junction characteristics between the two cases is explained on the basis of diffusion of $\mathrm{Cu}$ into the gold film to form an $\mathrm{AuCu}$ alloy in the first case, when the oxide layer is thermally formed on the Au film. the gold substrate, obtained after removal of the oxide layer, has a rose-gold color, clearly indicating the presence of an interfacial diffusion layer [11]. To confirm this, x-ray analysis of these substrates was also performed.

X-ray diffraction patterns were recorded in a Bragg-Brentano geometry in a Bruker D5005 diffractometer equipped with a Huber incident-beam monochromator and Braun PSD detector. Data collection was carried out at room temperature using $\mathrm{Cu} K \alpha 1$ radiation as the source. Evaluation of the measured data was done with the Bruker program EVA. All patterns are background-subtracted, meaning that the contribution of air scatter and possible $\mathrm{x}$-ray fluorescence radiation is subtracted.

The XRD pattern obtained from the cleaned gold substrate with the rose-gold surface is shown in Fig. 5 in black. The colored sticks correspond to the peak positions and intensities of $\mathrm{Au}$ and of the $\mathrm{AuCu}$ alloy taken from the ICDD pdf4 database [12]. The intensity versus $2 \theta$ values from the ICDD database correspond to the diffraction pattern from powdered samples with no preferential crystal orientation. It is possible to confirm the presence of a material if the position of the measured diffraction peaks in the $2 \theta$ axis matches with the position of the peaks in the database (the colored sticks). We see that in our measurement, the measured diffraction peak positions coincide with the peak positions of a $\mathrm{Au}-$ $\mathrm{Cu}$ alloy, in addition to gold and the silicon substrate.

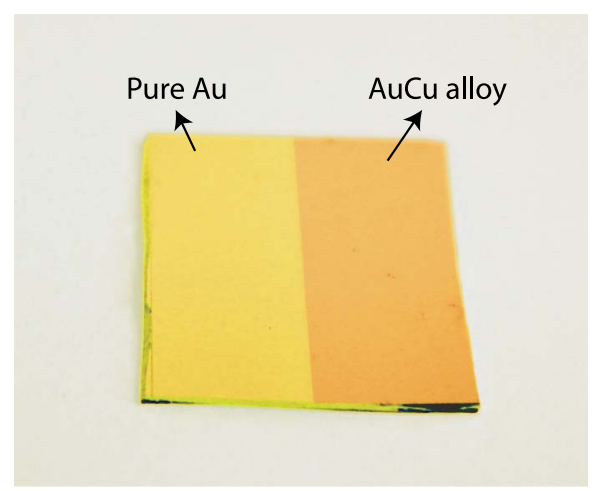

Fig. 4. Photograph of the $\mathrm{AuCu}$ alloyed sample surface. The right side of the sample was alloyed and has a slightly different rosegold color. The left side of the sample is a pure gold surface. The image was processed to obtain a better color contrast. 


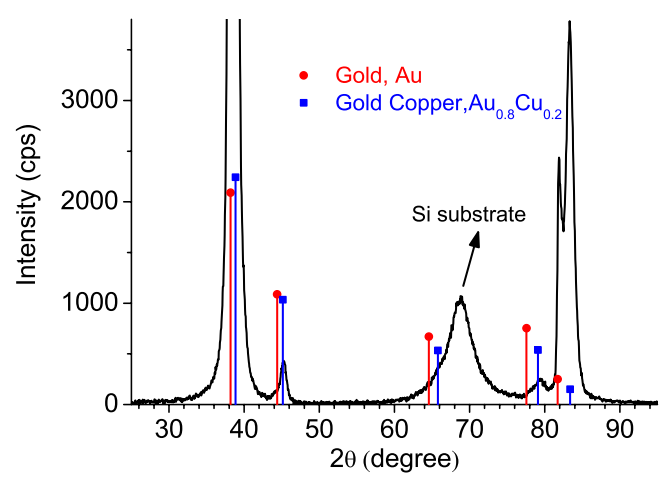

Fig. 5. XRD measurement of the gold substrates from which oxide was removed. No trace of oxide was found in the substrate.

The electron-beam-evaporated thin metal films usually have a preferred crystallographic orientation; hence, the ratio between the intensities of diffraction peaks do not correspond to that of the powdered sample given in the database. The measured x-ray diffraction peak at a $2 \theta$ value of $39^{\circ}$ suggests the presence of both $\mathrm{Au}$ and $\mathrm{AuCu}$ in the sample but around angles $45^{\circ}, 79^{\circ}$, and $83^{\circ}$. The measured peaks coincide better with the expected peak positions of the $\mathrm{AuCu}$ alloy. This proves that interdiffusion compounds of $\mathrm{Au}$ and $\mathrm{Cu}$ are formed at the interface. From the peaks around $38^{\circ}$ and $82^{\circ}$, it can be argued that some $\mathrm{Au}$ is also present in its pure form. From the XRD analysis, the alloy is estimated to have a composition of $A u_{0.8} \mathrm{Cu}_{0.2}$. However, since nitric acid can dissolve $\mathrm{Cu}$, we note that the process of removing the $\mathrm{Cu}_{2} \mathrm{O}$ layer could have caused some changes in the actual composition of the alloy layer. It can also be argued that the composition of the alloy may also vary in depth and laterally due to inhomogeneities in the diffusion. However, we do not see proof for this from the XRD analysis since the presence of several compositions would have given rise to more peaks in the XRD measurement. The main intention here is to show the presence of an interdiffusion layer and its importance in the $\mathrm{THz}$ generation process from our samples.

\section{Variable Angle Spectroscopic Ellipsometry Measurements}

Spectroscopic ellipsometry allows for accurate measurements of the optical properties of thin films. In this technique, the change in polarization state of the light reflected from the thin film substrate system is measured and is expressed by two values $(\Psi, \Delta)$ which are defined by the ellipsometric equation $[\underline{13}, \underline{14}]$

$$
e^{i \Delta} \tan (\Psi)=\rho=\frac{r_{p}}{r_{s}} .
$$

Here, $r_{p}$ and $r_{s}$ are the complex Fresnel reflection coefficients of the sample for $p$ - and $s$ - polarized incident light, respectively. $\Psi$ and $\Delta$ represent the angles determined from the amplitude ratio and phase difference between the reflected $p$ - and $s$ - polarized components, respectively. A model is constructed to calculate the values of $(\Psi, \Delta)$ using various parameters such as optical constants, layer thickness, and surface roughness. The generated and the experimentally determined values of $\Psi$ and $\Delta$ are compared and one or more model parameters are adjusted to fit the experimental data. This is done in an iterative manner until a good fit is reached between the measured and the experimental spectra. In variable angle spectroscopic ellipsometry, these values are measured over a wide range of wavelengths and angles of incidence which helps to minimize errors in extracting the material properties.

We performed ellipsometric measurements using a variable angle spectroscopic ellipsometer (VASE, J. A. Woollam Co.) in the wavelength region from 600 to $1600 \mathrm{~nm}$ and at different angles of incidence varying from $45^{\circ}$ to $60^{\circ}$. Initially, we fabricated individual thin films of $\mathrm{Au}$ and $\mathrm{Cu}_{2} \mathrm{O}$ on silicon wafers and measured the $(\Psi, \Delta)$ values of the light reflected from these samples. The refractive indices were obtained from the best fit model. The gold film was modeled to have a Drude-like conductivity. The frequency-dependent refractive indices of the gold film were measured because the properties of a thin film deposited by e-beam evaporation may be different from that of the bulk since they depend on the conditions of evaporation like the chamber pressure, rate of deposition, etc.

We then measured the $(\Psi, \Delta)$ values of the light reflected from a thin $\mathrm{Cu}_{2} \mathrm{O}$ film on $\mathrm{Au}$ formed by the thermal oxidation of copper. Based on the assumption that there is an interdiffusion layer of $\mathrm{AuCu}$ formed at the interface, we included this layer in the model. Hence, the model consists of the $\mathrm{AuCu}$ alloy film on gold (which was considered as the substrate) and the $\mathrm{Cu}_{2} \mathrm{O}$ layer with surface roughness. At the start of the fitting procedure, the thickness of the pure gold film and the $\mathrm{AuCu}$ alloy was set to 50 and $0 \mathrm{~nm}$, respectively, and was then allowed to vary during the iterations. The refractive index of $\mathrm{AuCu}$ was allowed to vary in the model, and the frequency-dependent refractive indices of the material were parameterized by means of B-splines which provide a numerical tool for data fitting without considering any physical model [15]. A B-spline consists of an array of control or "knot" points and a set of interpolating polynomial functions. The permittivity values of the material assumed by the model at the knot points are so as to generate ( $\Psi$, $\Delta$ ) values which match with the experimentally measured ones. The imaginary part of the frequencydependent dielectric permittivity of the material is modeled as the B-spline function, and the real part is calculated from the Kramers-Kronig relations. To make physical sense, the imaginary part of the refractive index $k$ is always forced to be positive. Measured optical constants of the evaporated $50 \mathrm{~nm} \mathrm{Au}$ film were used as the starting values for fitting that of the $\mathrm{AuCu}$ film. The frequencydependent refractive indices of the $\mathrm{Cu}_{2} \mathrm{O}$ thin film 

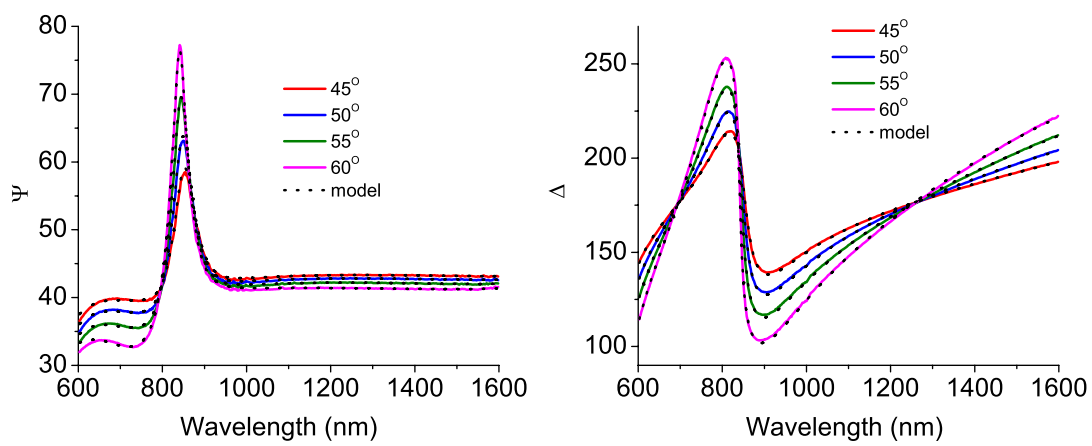

Fig. 6. $\Psi$ and $\Delta$ measurements of the light reflected from a $\mathrm{Cu}_{2} \mathrm{O}$ thin film on gold substrate (lines). The dots show the values of $\Psi$ and $\Delta$ calculated from the model used. Both $\Psi$ and $\Delta$ are plotted in units of degrees.

were also parameterized by means of B-splines. For $\mathrm{Cu}_{2} \mathrm{O}$, the initial values of the refractive index were taken from the measured values for a different oxide film on a silicon substrate.

The values of $\Psi$ and $\Delta$ for the sample from the experiment and the best-fit model are shown in Fig. 6. The colored solid lines correspond to the measured $\bar{\Psi}$ and $\Delta$ at different angles of incidence and the dashed black lines show the values calculated using the model. A good fit is obtained between the measurement and the model, as can be seen from the figure. The optical constants of the $\mathrm{Au}-\mathrm{Cu}$ alloy and $\mathrm{Cu}_{2} \mathrm{O}$ from the best-fit model are given in Fig. 7. For comparison, we also show the frequency-dependent refractive indices of $\mathrm{Au}$ in this figure. The thickness of the $\mathrm{Cu}_{2} \mathrm{O}$ thin film was around $232 \mathrm{~nm}$ with a roughness of $23 \mathrm{~nm}$. This agrees well with the average roughness $(\sim 21 \mathrm{~nm})$ measured using the AFM. Varying the thickness of the $\mathrm{AuCu}$ layer did not have any effect on the model-generated values of $(\Psi, \Delta)$. In other words, if we fit on the thickness of this layer, we get infinite uncertainty. This shows that the evaluation is mathematically not sensitive to the thickness change, because the lower interface of the $\mathrm{AuCu}$ layer cannot be seen. This indicates that the thickness of the $\mathrm{AuCu}$ film is larger than the penetration depth. The penetration depth of light of wavelength $700 \mathrm{~nm}$ in the $\mathrm{AuCu}$ thin film was calculated to be around $8 \mathrm{~nm}$. The importance of this fact is that our optical model can be much simpler since we can now use the $\mathrm{AuCu}$ layer as the substrate. Here, we also note that there could be an in-depth gradient in the formation of the $\mathrm{AuCu}$ alloy. This is not considered in our optical model in order to avoid creating a lot of fitting parameters. Some variations in the refractive indices of the $\mathrm{AuCu}$ alloy are observed for different $\mathrm{Cu}_{2} \mathrm{O}$ layer thickness, but overall, they are very similar. The oxidation time is the same for the samples of different $\mathrm{Cu}_{2} \mathrm{O}$ thickness; hence, the sample having a thicker $\mathrm{Cu}$ film gets more time for diffusion of $\mathrm{Cu}$ in $\mathrm{Au}$ before it is completely oxidized. This could probably explain the small differences in the refractive indices of $\mathrm{AuCu}$ alloy for different thicknesses of $\mathrm{Cu}_{2} \mathrm{O}$.

\section{Multilayer Reflection: Calculation and Experiment}

The $\mathrm{THz}$ emission from a $\mathrm{Cu}_{2} \mathrm{O} / \mathrm{Au}$ interface is proportional to the amount of laser power absorbed in the oxide film. At an optimum thickness, the $\mathrm{Cu}_{2} \mathrm{O}$ film can act as its own antireflection coating and hence increase the absorption inside the sample at a particular wavelength. Knowing the reflection spectra of the $\mathrm{Cu}_{2} \mathrm{O}$ thin films on oxide substrates, we can find the thickness at which the reflection minimum occurs at the pump laser wavelength, $800 \mathrm{~nm}$. Since we have extracted the frequencydependent refractive indices of the $\mathrm{Cu}_{2} \mathrm{O}$ and $\mathrm{AuCu}$ thin films, we can calculate the reflection spectra of the oxide films on the $\mathrm{AuCu}$ substrate for various thicknesses. We also experimentally measured the

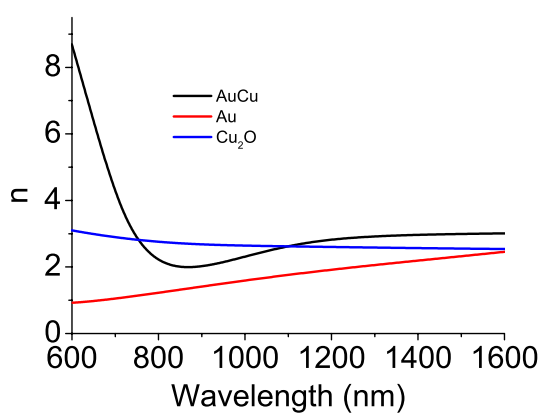

(a)

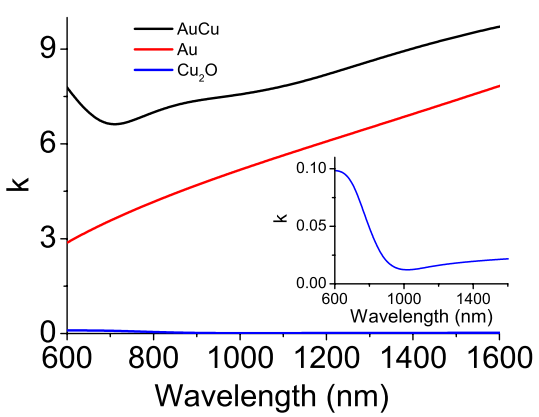

(b)

Fig. 7. Frequency-dependent real $(n)$ and imaginary $(k)$ parts of the refractive index of the $\mathrm{AuCu}$ interdiffusion layer, the $\mathrm{Cu}_{2} \mathrm{O}$ thin film, and the Au thin film, as obtained using the VASE software. The inset of (b) gives a closer look at the imaginary part of the refractive index of the $\mathrm{Cu}_{2} \mathrm{O}$ thin film. 


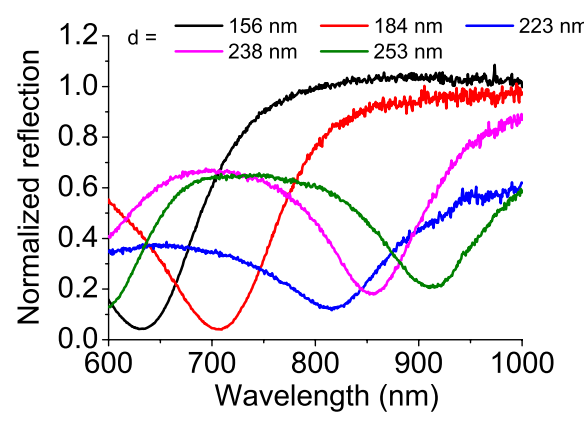

(a)

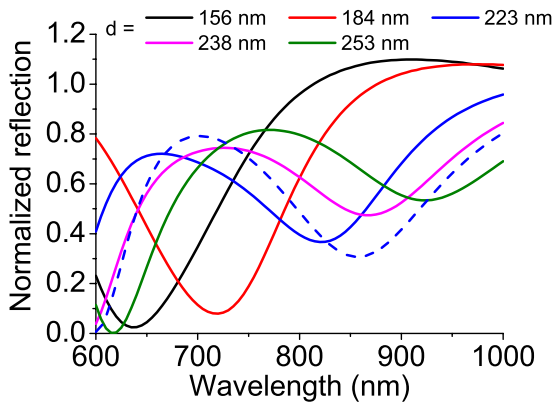

(b)

Fig. 8. White light reflection spectra from $\mathrm{Cu}_{2} \mathrm{O}$ layers of different thicknesses "d" on an AuCu substrate, normalized to the reflection from bare gold substrates. (a) Experimentally measured and (b) calculated from the analytical model using the complex refractive indices of the materials obtained from the ellipsometry analysis. The dashed blue line shows the calculated reflection spectrum of a $\mathrm{Cu}_{2} \mathrm{O}$ thin film of thickness $223 \mathrm{~nm}$ on pure $\mathrm{Au}$.

broadband reflection spectra of samples of five different $\mathrm{Cu}_{2} \mathrm{O}$ thicknesses on $\mathrm{AuCu}$ substrates to validate the complex refractive indices of the materials obtained from the ellipsometer analysis.

To measure the reflection spectrum, a Tungsten halogen lamp (HL 2000, Ocean Optics) was used as the broadband visible light source. The light from the source was passed through a polarizer and was incident upon the samples at $45^{\circ}$ angle of incidence. The spectrum of the reflected light was measured using an Ocean Optics spectrometer. The $\mathrm{Cu}_{2} \mathrm{O}$ layer thicknesses of the samples used in the experiment were estimated using the variable angle spectroscopic ellipsometer. The intensity of the light reflected from the oxide film on the gold substrate was measured as a function of wavelength and normalized to the reflection from a bare gold film. The reflectivity spectra thus obtained for five different $\mathrm{Cu}_{2} \mathrm{O}$ film thicknesses are plotted in Fig. 8(a). The calculation of the wavelength-dependent reflectivity, also normalized to the reflection from a bare gold film, was done with an analytical solution [16] which is suitable for multilayers containing strong absorbing elements such as metals. The calculated reflection spectra are shown in Fig. $8(\mathrm{~b})$. The multilayer stack considered for the reflection coefficient calculation consists of the silicon substrate, a $50 \mathrm{~nm}$ thick $\mathrm{Cr}$ film, $50 \mathrm{~nm} \mathrm{AuCu}$ layer, and the $\mathrm{Cu}_{2} \mathrm{O}$ film. To calculate the reflection spectra, we used the frequency-dependent complex refractive index of the materials, obtained from analyzing the ellipsometry measurements. We can see that there is good agreement between the calculated and the measured reflection spectra. The dashed blue line in Fig. 8(b) shows the calculated reflection spectrum of a sample with a $223 \mathrm{~nm}$ thick $\mathrm{Cu}_{2} \mathrm{O}$ film on a pure $\mathrm{Au}$ substrate, rather than on a $\mathrm{AuCu}$ alloy substrate. It can be seen that this does not match well with the experimentally measured spectrum for the same oxide thickness. In particular, the position of the calculated minimum at $\sim 855 \mathrm{~nm}$ is off compared to the measured one at $\sim 820 \mathrm{~nm}$. The thickness of the oxide layer of this sample was also measured independently, using a surface profilometer, to be $\sim 225 \mathrm{~nm}$ which is very close to the value of $223 \mathrm{~nm}$ extracted from the ellipsometer measurement. The minimum in the calculated reflection spectrum of this sample occurs at a different wavelength in the case of the $\mathrm{Au}-\mathrm{Cu}$ alloy instead of $\mathrm{Au}$ as the substrate for the $\mathrm{Cu}_{2} \mathrm{O}$ layer, since the dielectric function of $\mathrm{AuCu}$ is different compared to $\mathrm{Au}$. Hence, there is a difference in the phase change on reflection compared to that from $\mathrm{Au}$, leading to the shift in the calculated minimum in the reflection shown in Fig. 8(b). The real and imaginary parts of the frequency-dependent refractive indices of $\mathrm{Au}$ and $\mathrm{AuCu}$ are compared in Fig. 7. Good agreement between the measured and calculated reflection spectra is obtained only when the $\mathrm{AuCu}$ thin film is considered in the calculation, again supporting the presence of the interdiffusion $\mathrm{AuCu}$ alloy layer and validating the optical constants derived from the ellipsometer measurements. The slight discrepancies between the calculated and measured reflection spectra could be due to a combination of several issues. The reflection calculation, for example, ignores the roughness of the oxide film in the calculation, the lateral inhomogeneities in the refractive index of the $\mathrm{Cu}_{2} \mathrm{O}$ layer, and experimental errors in determining the angle of incidence. It can also be seen from the calculated and reflected spectra that an oxide film of $\sim 223 \mathrm{~nm}$ thickness is well suited for $\mathrm{THz}$ emission applications since it has a very low reflection coefficient at $800 \mathrm{~nm}$ which is the pump laser wavelength used in $\mathrm{THz}$ generation experiments.

\section{Conclusions}

We have shown that interfaces between gold and thermal $\mathrm{Cu}_{2} \mathrm{O}$, which emit $\mathrm{THz}$ radiation when illuminated with ultrafast femtosecond lasers, are in fact $\mathrm{AuCu} / \mathrm{Cu}_{2} \mathrm{O}$ interfaces due to the formation of a thermal-diffusion-induced alloy of $\mathrm{AuCu}$ at the interface. This alloy plays an important role in the $\mathrm{THz}$ emission by enabling the formation of a Schottky-barrier-like junction. The presence of the alloy layer has been confirmed using x-ray diffraction measurements, ellipsometry, and visual inspection. The optical properties of the alloy and $\mathrm{Cu}_{2} \mathrm{O}$ thin films have been characterized using variable angle 
spectroscopic ellipsometry and were used to calculate the reflection spectra which agree well with the measurements.

P. C. M. P. and A. J. L. A. acknowledge the financial support from the Nederlandse Organisatie voor Wetenschappelijk Onderzoek (NWO) and the Stichting voor Technische Wetenschappen (STW) in the form of VICI and VENI grants, respectively. We acknowledge the help of Peter Swart, DIMES technology center, TU Delft, for his help in measuring the I-V curve.

\section{References}

1. X. Zhang, J. T. Darrow, B. B. Hu, D. H. Auston, M. T. Schmidt, P. Tham, and E. S. Yang, "Optically induced electromagnetic radiation from semiconductor surfaces," Appl. Phys. Lett. 56, 2228-2230 (1990).

2. Y. Jin, X. F. Ma, G. A. Wagoner, M. Alexander, and X. C. Zhang, "Anomalous optically generated $\mathrm{THz}$ beams from metal/GaAs interfaces," Appl. Phys. Lett. 65, 682-684 (1994).

3. G. Ramakrishnan, G. K. P. Ramanandan, A. J. L. Adam, M. $\mathrm{Xu}, \mathrm{N}$. Kumar, R. W. A. Hendrikx, and P. C. M. Planken, "Enhanced terahertz emission by coherent optical absorption in ultrathin semiconductor films on metals," Opt. Express 21, 16784-16798 (2013).

4. W. Wan, Y. Chong, L. Ge, H. Noh, A. D. Stone, and H. Cao, "Time-reversed lasing and interferometric control of absorption," Science 331, 889-892 (2011).

5. L. C. Olsen, R. C. Bohara, and M. W. Urie, "Explanation for low-efficiency $\mathrm{Cu}_{2} \mathrm{O}$ Schottky-barrier solar cells," Appl. Phys. Lett. 34, 47-49 (1979).
6. G. Papadimitropoulos, N. Vourdas, V. E. Vamvakas, and D. Davazoglou, "Deposition and characterization of copper oxide thin films," J. Phys. 10, 182-185 (2005).

7. G. K. P. Ramanandan, G. Ramakrishnan, and P. C. M. Planken, "Oxidation kinetics of nanoscale copper films studied by terahertz transmission spectroscopy," J. Appl. Phys. 111, 123517 (2012).

8. N. C. J. van der Valk, T. Wenckebach, and P. C. M. Planken, "Full mathematical description of electro-optic detection in optically isotropic crystals," J. Opt. Soc. Am. B 21, 622-631 (2004).

9. Y. P. Yang, W. C. Wang, Z. W. Zhang, L. L. Zhang, and C. L. Zhang, "Dielectric and lattice vibrational spectra of $\mathrm{Cu}_{2} \mathrm{O}$ hollow spheres in the range of 1-10 THz," J. Phys. Chem. C 115, 10333-10337 (2011).

10. R. Morrish, K. Dorame, and A. Muscat, "Formation of nanoporous $\mathrm{Au}$ by dealloying $\mathrm{AuCu}$ thin films in $\mathrm{HNO}_{3}$," Scr. Mater. 64, 856-859 (2011).

11. C. Cretu and E. van der Lingen, "Coloured gold alloys," Gold Bull. 32, 115-126 (1999).

12. J. Faber and T. Fawcett, "The powder diffraction file: present and future," Acta Crystallogr. Sect. B 58, 325-332 (2002).

13. J. A. Woollam, B. D. Johs, C. M. Herzinger, J. N. Hilfiker, R. A. Synowicki, and C. L. Bungay, "Overview of variable-angle spectroscopic ellipsometry (VASE): I. Basic theory and typical applications," Proc. SPIE CR75, 3-28 (1999).

14. H. Fujiwara, Spectroscopic Ellipsometry: Principles and Applications (Wiley, 2007).

15. J. W. Weber, T. A. R. Hansen, M. C. M. Van de Sanden, and R. Engeln, "B-spline parametrization of the dielectric function applied to spectroscopic ellipsometry on amorphous carbon," J. Appl. Phys. 106, 123503 (2009).

16. O. El Gawhary, M. C. Dheur, S. F. Pereira, and J. J. M. Braat, "Extension of the classical Fabry-Perot formula to 1D multilayered structures," Appl. Phys. B 111, 637-645 (2013). 